\title{
PROMOCIÓN DEL DESARROLLO COMO PRÁCTICA DE CRIANZA DE LAS MADRES ADOLESCENTES*
}

\section{DEVELOPMENT PROMOTION AS REARING PRACTICE OF TEENAGE MOTHERS}

\author{
Nadia Milena Henao-García** \\ MÓNICA MARÍA VILLA-GIL**
}

\section{Resumen}

Objetivo. Mostrar los resultados de la investigación "Prácticas de crianza en madres adolescentes” en la categoría Desarrollo. Caracterizar las prácticas de crianza que promueven el desarrollo infantil, y que a su vez propician bienestar y satisfacción con la vida. Metodología. El diseño metodológico es holístico-descriptivo. Resultados. Se encontraron tres subcategorías: la cognitiva, en la cual las madres reconocen la implementación de juegos y ejercicios matemáticos; la corporal, ya que promueven la estimulación física y la participación en los programas de control y desarrollo, y la estética, ya que propician espacios al niño para que valore sus formas de sentir e interactuar. Conclusión. La mujer en su rol de madre es la que facilita el desarrollo de los niños; sin embargo, es una tarea que la sociedad le atribuye en medio de un contexto que cada vez le asigna más tareas en la crianza, pero que la deja más sola para asumirlas.

Palabras clave: madres adolescentes, pautas y prácticas de crianza, desarrollo infantil, dimensiones del desarrollo.

\begin{abstract}
Objective. This paper shows the results of the research "Practices of Child Rearing in Teenage Mothers" in the Development category, characterizing child rearing practices that promote child development which, in turn, promote wellbeing and satisfaction with life. Methodology. The methodological design is holistic-descriptive. Results. Three subcategories were found: cognitive, in which mothers recognize the implementation of games and mathematical exercises; corporal, since it promotes physical stimulation and participation in control and development programs; and, aesthetic since it provides spaces for the child to value their ways of feeling and interacting. Conclusion. Women, in their role as mothers, become facilitators of children's development. However, it is a task that society attributes to women, in a context that increasingly assigns them more tasks in child parenting, but leaves them more lonely to assume such tasks.

\footnotetext{
* El presente artículo fue escrito con base en los resultados del proyecto "Prácticas de crianza en madres adolescentes", desarrollado entre los años 2016 y 2017, el cual se centró en la caracterización de las prácticas de crianza que implementan las madres adolescentes para promover el desarrollo.

***Tecnológico de Antioquia - I.U. Medellín, Colombia. E-mail: nmhenaog@tdea.edu.co -

(D) orcid.org/0000-0002-1826-7024. Google Scholar

***Fundación Hermanos de los Desvalidos. Medellín, Colombia. E-mail: mmvillagil@yahoo.es -

(1) orcid.org/0000-0002-1170-1166. Google Scholar
} 
Key words: teenage mothers, patterns and practices of child rearing, child development, development dimensions.

\section{Introducción}

La adolescencia es una etapa del ciclo vital humano que comprende las personas entre los 12 y 18 años de edad (Ley 1098 de 2006) y, aunque no hay un consenso en cuanto al rango de edad, diversos autores coinciden en afirmar que es un momento de la vida caracterizado por cambios biológicos, fisiológicos, psicológicos y sociales, cuyo desarrollo se ve afectado por aspectos externos a ellos, es decir, por cambios o condiciones socioeconómicas, culturales, sociales, educativas, familiares y personales (UNICEF-Centro de Investigaciones Innocenti, 2006).

Una de las realidades que afectan el pleno desarrollo de las adolescentes es el embarazo y la maternidad a temprana edad. Esto se presenta por múltiples razones, entre ellas el inicio de relaciones sexuales a edades tempranas, las condiciones de pobreza, el no ingreso al sistema educativo, la ausencia de educación sexual y reproductiva, el desconocimiento en el uso de métodos de control natal. Se ha conocido que en algunos de los casos las adolescentes deciden ser madres con el fin de salir de sus casas para conformar otra "familia", por motivos relacionados con la invisibilización que sufren en sus hogares, por falta de apoyo para estudiar, por violencia intrafamiliar, o simplemente porque se tiene el imaginario en algunas culturas de que el tener un hijo hace permanecer vinculada sentimental y económicamente a la pareja.

La Encuesta Nacional de Demografía y Salud del 2010, expresa que "si bien las tasas de fecundidad de las adolescentes han descendido en la mayoría de los países en vías de desarrollo durante los últimos 20 años, la conducta reproductiva de este grupo constituye gran preocupación" (Profamilia, 2010, p. 121). Según esta misma encuesta, en Colombia una de cada cinco mujeres de 15 a 19 años ha estado alguna vez embarazada: 16\% ya son madres y 4\% están esperando su primer hijo. Por su parte la CEPAL, Naciones Unidas y OIJ (2007) afirman que:

la maternidad adolescente fuera de uniones o matrimonio se da especialmente en el grupo de 15 a 17 años, perteneciente a los sectores más pobres y mayormente expuestos a procesos de exclusión temprana (del sistema educativo y de inserción precaria y temprana en el mercado de trabajo). (p. 86) 
Promoción del desarrollo como práctica de crianza de las madres adolescente

Ante el nacimiento de sus hijos, las adolescentes se ven enfrentadas a asumir un rol materno para el que generalmente no se prepararon, lo que conlleva a cambios de hábitos propios de la etapa del ciclo vital que vivencian, así como también tendrán que crear o recrear unas prácticas de crianza con las cuales deberán orientar el desarrollo del niño. Dichas prácticas de crianza han de entenderse como las "acciones que se orientan a garantizar la supervivencia del infante, a favorecer su crecimiento y desarrollo psicosocial, y a facilitar el aprendizaje de conocimientos que permitan al niño reconocer e interpretar el entorno que le rodea" (Aguirre, 2000, p. 28).

Si se parte del supuesto de que la madre que ejecuta estas acciones es una adolescente, que ejerce un rol materno y por ende de crianza, es necesario preguntarse: ¿cuáles serán las pautas y prácticas que implementa para alcanzar el desarrollo de sus hijos durante la crianza? Acciones que en muchas oportunidades se ven afectadas por condiciones socioeconómicas, culturales, educativas, familiares y personales. Lo cual trae como consecuencia la delegación del rol materno a terceras personas - abuela materna o paterna, vecinas, entre otrasque se convierten en cuidadores de sus hijos, situación que puede afectar de manera positiva o negativa el desarrollo integral de los niños.

A partir del supuesto o hipótesis descrito anteriormente y teniendo claro que la población objeto de estudio está constituida por las madres adolescentes, el presente proyecto de investigación se justificó por un lado, en que es evidente el aumento de los embarazos en la adolescencia, lo que lleva a asumir un rol materno mucho más temprano, en una etapa de la vida en la que aún están avanzando en su propio desarrollo físico e identidad, con lo que las prácticas de crianza pueden verse afectadas. Y del otro, en la medida en que se reconoce que estas prácticas implementadas en los hogares inciden en el proceso de desarrollo, bienestar e integración a la vida social de los niños, en tanto son acciones conducentes al afianzamiento de la personalidad, la conducta, la relación consigo mismo y con los demás.

En tal sentido, el objetivo desarrollado en este artículo buscó caracterizar las prácticas de crianza de las madres adolescentes dirigidas a promover el desarrollo integral de los niños.

\section{Referente teórico y conceptual}

\section{Madres adolescentes}

La maternidad es una categoría socialmente construida que configura el rol de la mujer en las relaciones parentofiliales (Alvear y Erazo, 2003). Es una construcción histórico-social que supera el ámbito de lo biológico y se carga de un amplio contenido simbólico asociado por tradición al carácter protector, afectivo, al cuidado y la crianza desempeñada por las mujeres en sus grupos familiares (Ramírez y Barrios, 2016). 
La gestación o embarazo en edades tempranas hace referencia al concepto de 'adolescencia' como se planteó anteriormente. Para el UNICEF (2011), la población adolescente está comprendida entre los 10 y 19 años y se subdivide en tres etapas: adolescencia temprana entre los 10 y 13, media entre los 14 y 16, y tardía entre los 17 y 19 años.

El UNFPA (2013) en su informe sobre maternidad en niñez plantea el tema como un problema mundial, en donde las menores de 19 años en embarazo o ejerciendo la maternidad temprana representan la perpetuación de la pobreza, la marginación, dependencia y exclusión, esto conlleva a que se afecte negativamente su salud, su educación y su potencial de obtener ingresos. Resaltando alrededor del asunto la construcción simbólica y cultural en donde los procesos de socialización y el acompañamiento de los padres cumplen un papel fundamental y directo sobre la vida de las adolescentes, en la medida en que pueden transformar modelos de desigualdad de género, dependencia y baja autoestima por prácticas de crianza y procesos de socialización que fortalezcan una conciencia de equidad y oportunidades sin discriminación de género (UNFPA, 2013).

\section{Prácticas de crianza}

El concepto de prácticas de crianza se asume desde los planteamientos de Aguirre (2000), el cual expresa que son acciones que los padres o cuidadores desarrollan para garantizar el crecimiento y desarrollo psicológico y social de los niños, en favor de su supervivencia y aprendizajes. Dichas acciones se ven influenciadas por factores socioeconómicos, culturales, sociales, educativos, familiares y personales, de los padres o cuidadores a cargo de la crianza de los niños.

Las prácticas de crianza, por tanto, están estrechamente ligadas con el proceso de socialización necesario en todos los grupos humanos, a fin de garantizar la supervivencia y la integración a la vida social. Estas acciones, que hacen los padres o adultos a cargo de los niños, están relacionadas con las pautas (normatividad) y las creencias (explicaciones) enfocadas a la construcción humana del nuevo ser. Para su ejercicio son necesarios un conjunto de recursos y materiales humanos y financieros que pueden influir en el desarrollo de la persona (Torres, 2009).

Las prácticas tales como alimentar, bañar, enseñar y dormir a los niños, pueden ser comunes a todas las sociedades, pero también es cierto que estas pueden variar de un lugar a otro, pues dependen de las pautas, es decir, de los patrones, normas y costumbres, como de las creencias relacionadas con los valores, mitos y prejuicios que los padres o adultos tienen frente a la crianza, y que se transmiten generacionalmente. Frente a esto Myers (como se citó en Aguirre, 2000) plantea que "si se las quiere definir y distinguir... es necesario diferenciar lo que se hace de cómo se hace” (p. 33). 
Promoción del desarrollo como práctica de crianza de las madres adolescente

\section{Pautas de crianza}

Las pautas de crianza "se relaciona con el qué se debe hacer y se refiere a lo esperado en la conducción de las acciones de los niños. Es el vínculo directo con las determinaciones culturales propias del grupo de referencia” (Aguirre, 2000, p. 29). Las pautas, por tanto, se convierten en cánones normativos que son aceptados y generalmente legitimados por las sociedades.

Las personas - padres o cuidadores- responsables de la crianza de los niños en sus primeros meses y años de vida, pueden seguir o no dichos patrones o costumbres, todo depende de las circunstancias de índole económico, social, cultural, personal, educativo y familiar que rodean al niño en su proceso de crecimiento y desarrollo, así como también de las diferentes creencias y conocimientos de las personas a cargo de la crianza de los niños (Aguirre, 2000).

Cada cultura, por lo tanto, es la encargada de promover las pautas de crianza con el fin de transmitir generacionalmente valores y costumbres a cada sociedad, dotando de sentido las interacciones sociales de cada sujeto. Por eso, cuando se habla de pautas, es necesario remitirse a la historia y a las construcciones culturales de cada sociedad a fin de comprender las actitudes que se han tenido respecto a la infancia, estipulando el cómo deben ser educados los niños según las características de cada contexto social, cultural y familiar en los que se encuentran inmersos (Henao y Villa, 2017).

\section{Desarrollo infantil}

Se ha visualizado en el desarrollo infantil la esperanza de contar con generaciones futuras capaces de ejercer su ciudadanía, que exijan sus derechos y puedan lograr mayores niveles de equidad y bienestar para todos. Pero también es claro que estas condiciones pueden alcanzarse siempre que se den las garantías necesarias para lograrlo. Esto quiere decir que en entornos de deprivación afectiva, maltrato, enfermedad y pobreza, difícilmente la sociedad podrá acercarse a las expectativas planteadas.

Para Martínez (2014), “el desarrollo de los niños corresponde a una interacción dinámica que refleja las características esenciales de un ser vivo y está influenciado por la naturaleza y la crianza” (p. 124). Señala el autor que la crianza está determinada por la familia y la comunidad, el tipo de cuidados, valores, prácticas y creencias que toman de la cultura a la que pertenecen; a su vez, la influencia de la naturaleza está dada por la relación que el niño establece con el medio ambiente y el medio ambiente con el niño; bajo esta experiencia el desarrollo es moldeado de manera única e irrepetible en cada ser humano. Lo anterior quiere decir que los componentes ambientales-biológicos están integrados produciendo de manera no lineal o estática condiciones para el desarrollo de los niños. 
Siguiendo la misma línea, Restrepo (2015) ha dicho que el desarrollo infantil busca el aumento gradual durante el crecimiento de los niños, de habilidades que le permitan adaptarse a las condiciones cambiantes de su entorno, superen la inmadurez y la dependencia y sean capaces de entablar relaciones con otros. En lo que interviene una condición biológica determinada por la herencia genética, por un componente psicológico y uno social, siendo este último en que los niños a través de las interacciones sociales logran adaptarse a su medio.

En tal sentido, se da en los niños un primer desarrollo en las estructuras biológicas, con la conformación de las redes neurales y cerebrales que permiten los procesos de aprendizaje y representación mental del espacio en que se habita. Posteriormente aparece el desarrollo social, con las interacciones sociales que le abren paso a la inserción a la cultura.

El desarrollo se concentra en satisfacer todas las necesidades humanas fundamentales, las cuales están ligadas a las dimensiones del desarrollo, como un elemento de integralidad. Las dimensiones a las cuales se hace referencia son: dimensión corporal, dimensión socioemocional, dimensión cognoscitiva, dimensión comunicativa, dimensión ética y dimensión estética.

\section{Dimensión corporal}

Hace referencia a los procesos de supervivencia, crecimiento y desarrollo, estrechamente vinculados con el mundo físico, además implica el reconocimiento del propio cuerpo y las posibilidades de relación con el mundo. Se relaciona con la salud y nutrición; se inicia con el desarrollo de habilidades sensoriales y psicomotrices y la identificación de género (Amar, 2015).

\section{Dimensión socioemocional}

Mediante esta dimensión adquirimos capacidades para tener cierto manejo de emociones como la ira, el miedo, conocer lo profundo de nuestra intimidad y regular las relaciones interpersonales (Amar, 2015). Es fundamental desde el nacimiento construir un ambiente agradable, donde se fortalezca su desarrollo afectivo y se les brinde el espacio para que puedan expresar sus sentimientos.

\section{Dimensión cognoscitiva}

Construcción de conocimiento por parte del ser humano (Amar, 2015). Es indispensable propiciar ambientes donde los niños puedan explorar, crear e imaginar y así construir conocimiento a partir de sus experiencias. 
Promoción del desarrollo como práctica de crianza de las madres adolescente

\section{Dimensión comunicativa}

Su núcleo fundamental es el lenguaje, que constituye un papel fundamental en el proceso de construcción de signos y códigos que cumplen una función representativa del mundo simbólico y material; las primeras experiencias establecidas con los cuidadores son vitales para el desarrollo de habilidades comunicativas (Amar, 2015). Es importante que los adultos que están alrededor de los ninos utilicen un lenguaje adecuado sin usar palabras soeces porque todo lo que los parvularios escuchen lo tomarán de referencia.

\section{Dimensión ética}

Esta dimensión fomenta y nutre las capacidades de ser y actuar libremente y al mismo tiempo respetar los ordenamientos que hacen posible una vida en sociedad, promoviendo el desarrollo de capacidades humanas para percibir y vivir derechos y deberes, así mismo la forma en que el colectivo social puede enseñar a las personas desde los primeros años de su vida a vivir con valores que les ayuden a la formación de su identidad y respetar a los demás (Amar, 2015). Durante los primeros años de vida se deben tener unas normas que posibiliten que los niños se adapten a diferentes situaciones, que aprendar a convivir con los demás y conozcan lo que es bueno y lo que es malo.

\section{Dimensión estética}

Se refiere a la satisfacción y el goce como experiencias humanas fundamentales. Es un espacio de desarrollo de la capacidad de creación de lo bello y de valores estéticos. El cultivo de esta dimensión genera disfrute y seguridad psicológica para la exploración (Amar, 2015). Es importante que los niños interactúen con sus pares y a partir de ellos comiencen a desarrollar todas sus capacidades.

Estas dimensiones se construyen desde los primeros meses de vida. Los niños van adquiriendo habilidades con ayuda de sus padres, mostrando reacciones con algunos estímulos que reciben para hablar, gatear, caminar y generar su autonomía y su relación con el entorno. Desde su nacimiento es indispensable que estos cuenten con experiencias emocionales, cognitivas y sociales buenas, para lograr formar personas capaces de desenvolverse mejor en la sociedad. También es importante la ayuda comprensiva de los padres, que permita al párvulo expresar sus sentimientos, pensamientos, entre otros, y así facilitar su adaptación a los diferentes cambios. 


\section{Materiales y Métodos}

Con miras a dar respuesta al interrogante planteado y cumplimiento al objetivo de investigación, relacionado con caracterizar las prácticas de crianza que implementan las madres adolescentes que participan en los programas académicos del Tecnológico de Antioquia, para promover el desarrollo de sus hijos, el proyecto se enmarcó en las investigaciones holísticas, las cuales surgen como una necesidad de proporcionar criterios de apertura a las ciencias sociales e integrar los paradigmas cuantitativo y cualitativo para una mayor comprensión de los fenómenos; lo que no quiere decir que sea un enfoque ecléctico es un planteamiento sistemático y organizado, con fases que hacen posible avanzar en la construcción de conocimiento social; "otro aporte que proporciona la investigación holística consiste en propiciar una mayor trascendencia de lo científico a otras áreas de lo humano y lo social” (Hurtado de Barrera, 2000, p. 30).

El tipo de investigación fue descriptivo, el cual según Hurtado de Barrera (2000) "consiste en la caracterización de un hecho, fenómeno, individuo o grupo, con el fin de establecer su estructura o comportamiento" (p. 630). En el caso de este estudio, se caracterizaron las prácticas de crianza como fenómeno, con un grupo poblacional específico: madres adolescentes participantes en los programas académicos del Tecnológico de Antioquia - Institución Universitaria (Henao y Villa, 2017).

Ander-Egg (1987) postula que la investigación descriptiva, además de caracterizar eventos o situaciones concretas indicando rasgos particulares, se constituye en un punto de partida para realizar estudios de mayor profundidad y avanzar a niveles de mayor complejidad como el comparativo, explicativo, proyectivo y evaluativo. Es intención del proyecto de investigación proponer, con base en los resultados obtenidos del presente estudio, la realización de otra fase de la investigación que se enfoque en el diseño e implementación de una propuesta formativa para madres adolescentes, que les permita asumir la crianza de sus hijos de forma asertiva desde la comprensión de sus acciones y el impacto en el desarrollo de sus hijos. Igualmente, los resultados del estudio podrán ser utilizados por los estudiantes de pregrado o posgrado de la IES para profundizar en asuntos como los patrones, las creencias o impacto de las prácticas de crianza, o comprender las relaciones, actitudes y roles de las madres adolescentes.

En la investigación holística, la unidad de estudio hace referencia al contexto, sujeto o entidad poseedores de las características, cualidades o categorías que se desean estudiar. En este estudio serán las prácticas de crianza, entendidas como las acciones concretas que las madres adolescentes llevan a cabo con el propósito de orientar la crianza de los niños, en aras del desarrollo, bienestar e integración de ellos a la vida social.

La población estuvo constituida por madres adolescentes que participan en los programas académicos del Tecnológico de Antioquia, teniendo en cuenta los siguientes criterios de 
Promoción del desarrollo como práctica de crianza de las madres adolescente

inclusión: ser madre antes de los 18 años, tener un vínculo con la IES, y que el hijo o hijos no superaran los 6 años de edad.

La técnica utilizada para el proceso de recolección de datos fue la encuesta. La encuesta a 38 madres adolescentes, permitió recoger "las declaraciones emitidas por una muestra representativa de una población concreta y que nos permite conocer sus opiniones, actitudes, creencias, valoraciones subjetivas, etc.” (García y Quintanal, 2007, p. 1). En el caso de este estudio se recogieron las declaraciones emitidas por las madres adolescentes respecto a las acciones que realizan para promover el desarrollo de sus hijos.

El instrumento utilizado fue el cuestionario, el cual consistió en un conjunto de preguntas, usualmente de varios tipos, cuya finalidad fue "obtener, de manera sistemática y ordenada, información acerca de la población con la que se trabaja, sobre las variables objeto de la investigación” (García, 2003, p. 2).

Para el proceso de registro y sistematización de la información se incorporó la mirada cuantitativa, pues el estudio, partiendo de un constructo teórico, se encaminó al análisis de datos a partir de la exploración, el descubrimiento y la descripción en torno al análisis de una realidad objetiva. Se utilizó una base de datos en Excel, en la que se registraron las respuestas. En una segunda fase, luego de que se recogió el total de la información en la población considerada para el estudio, se hizo un proceso de depuración de cada base de datos con el fin de minimizar los errores que surgen en el vaciado de los datos.

El análisis de la información fue descriptivo, con la intencionalidad de caracterizar un objeto de estudio, señalar sus características y propiedades. De acuerdo a la información recogida se está efectuando análisis con datos desagregados según variables y categorías necesarias para el estudio.

\section{Resultados}

Las madres adolescentes, respecto a las prácticas de crianza enfocadas al desarrollo de sus hijos, manifiestan acciones conducentes a promover la construcción de dimensiones del desarrollo como la cognitiva, corporal y estética. Tales acciones se evidencian a continuación, a partir de una serie de preguntas que permitieron develar acciones puntuales.

\section{Cognitivo}

La madre adolescente acompaña el desarrollo cognitivo de sus hijos a partir de acciones concretas, mediadas por la estimulación y actividades que favorecen la movilización de aprendizajes. Respecto a las actividades que las madres utilizan para estimular el desarrollo 
del niño, se encuentra que el 21\% de las madres estimulan el desarrollo cognitivo de sus hijos con juegos, música, lectura, dibujos y cantos y el $18 \%$, a parte de las opciones dadas, plantean otras formas de estimulación como: videos, televisión, salidas a parques, practicando deportes, entre otras. Las demás seleccionaron entre una y cuatro respuestas, lo que evidencia que ellas implementan diversas actividades para estimular el desarrollo de la dimensión cognitiva de sus hijos. Es importante resaltar que ninguna marcó la opción 6, la cual hace referencia a la NO implementación de actividades para la estimulación del desarrollo (ver Figura 1), reconociendo la importancia de la estimulación a partir de diversas actividades, para el desarrollo cognitivo desde los primeros años de vida.

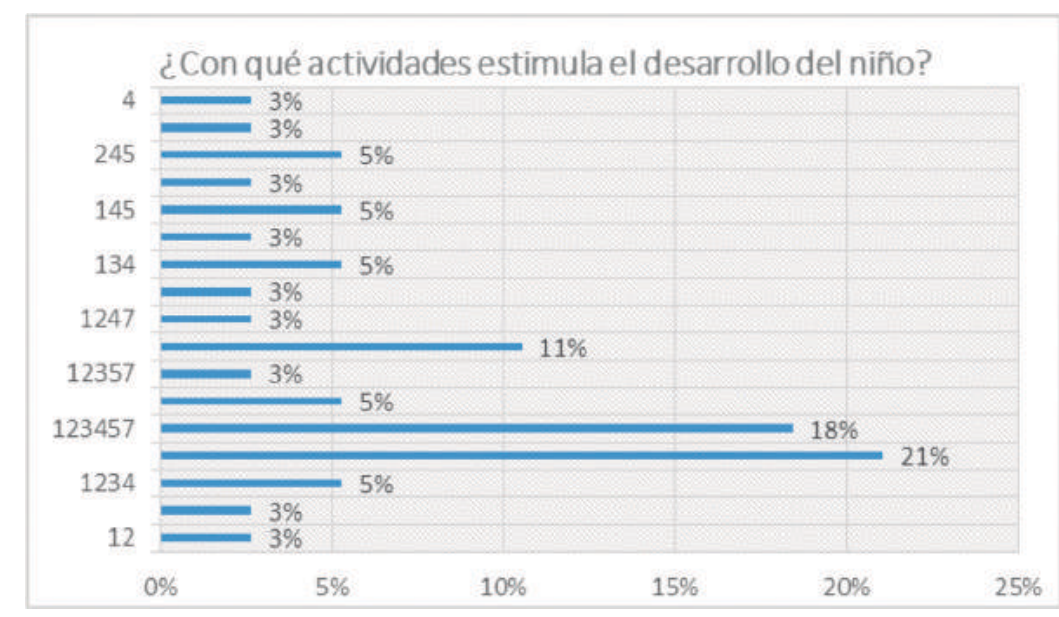

Fuente: elaboración propia.

Figura 1. Actividades para estimular el desarrollo del niño. (1) juegos, (2) música, (3) lectura, (4) dibujos, (5) cantos, (6) ninguno, (7) otros.

Lo anterior se relaciona con las acciones enfocadas a la enseñanza de algunos temas mediados por el juego. Se encontró que el $11 \%$ de las madres manifiestan enseñar a sus hijos: nombres de lugares, animales, objetos, personas, partes del cuerpo, nociones matemáticas, leer y escribir, memorizar, ordenar y clasificar objetos y a resolver problemas; las demás marcaron entre una y siete opciones de respuesta. Entre algunas otras opciones se encuentran: valores, colores, números e historia del país. Lo anterior evidencia que, en su mayoría, las madres a las cuales se les realizó la encuesta se ocupan por enseñarles a sus hijos nociones y elementos importantes para el desarrollo de la dimensión cognitiva de los niños que serán fundamentales para los procesos de escolarización posteriores (ver Figura 2). 
Promoción del desarrollo como práctica de crianza de las madres adolescente

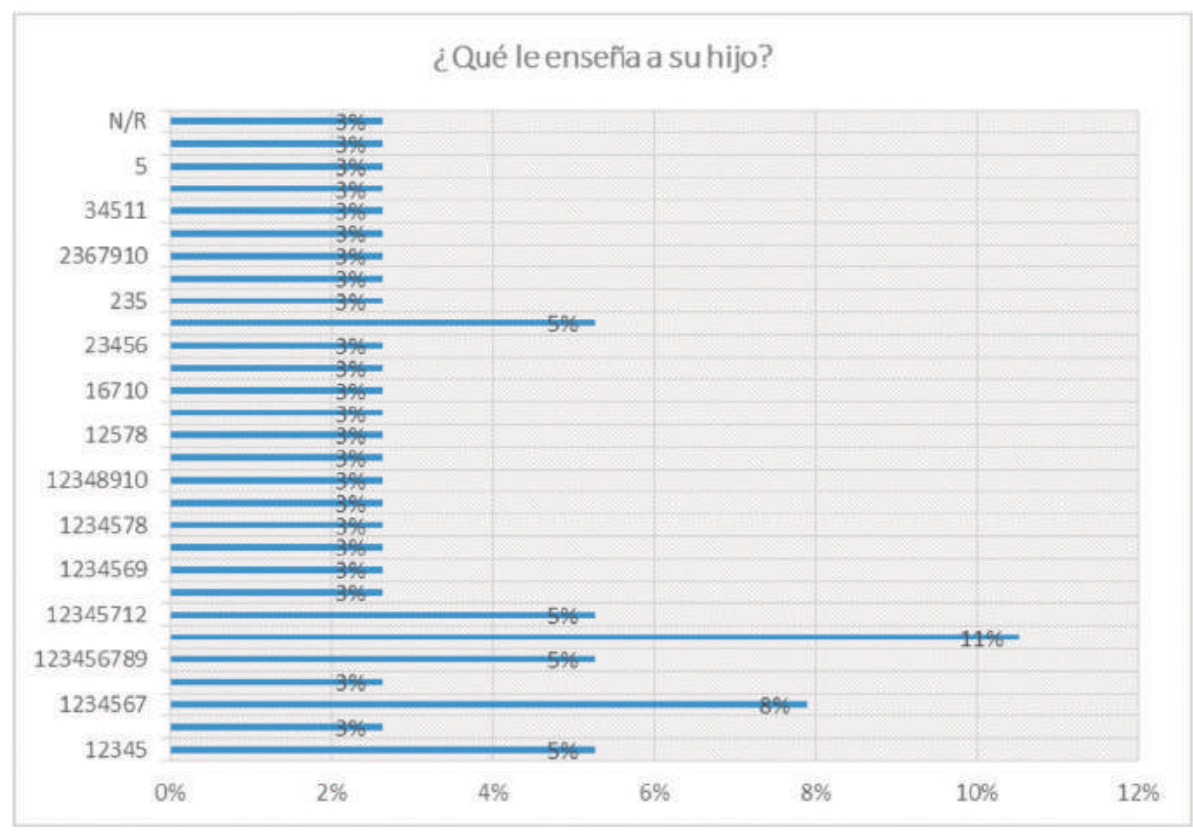

Fuente: elaboración propia.

Figura 2. Acciones enfocadas al proceso de enseñanza. (1) nombres de lugares, (2) animales, (3) objetos, (4) personas, (5) partes del cuerpo, (6) nociones matemáticas, (7) leer y escribir, (8) memorizar, (9) ordenar y clasificar objetos, (10) resolver problemas, (11) otras.

Finalmente se indagó por el acompañamiento que realizan las madres a las actividades que le ponen en el jardín infantil o en la escuela, teniendo en cuenta que muchos de los niños aún no han ingresado al sistema de atención integral a la primera infancia o a la educación formal. Respecto a esto, se encuentra que el 68\% Sí acompañan dicho proceso por medio de explicaciones, repasos, guiándolos en la actividad, entre otros, manifestando que, aunque acompañan, permiten que ellos sean autónomos; el 29\% NO realizan dicho acompañamiento y el 3\% restante no respondieron a la pregunta formulada. Es importante aclarar que el 29\% de las madres que manifiestan no acompañar a sus hijos en las actividades del jardín infantil o la escuela, lo justifican en la medida en que los niños por su edad aún no ingresan a dichos espacios, y una de ellas plantea que no realiza el acompañamiento por falta de tiempo (ver Figura 3). 


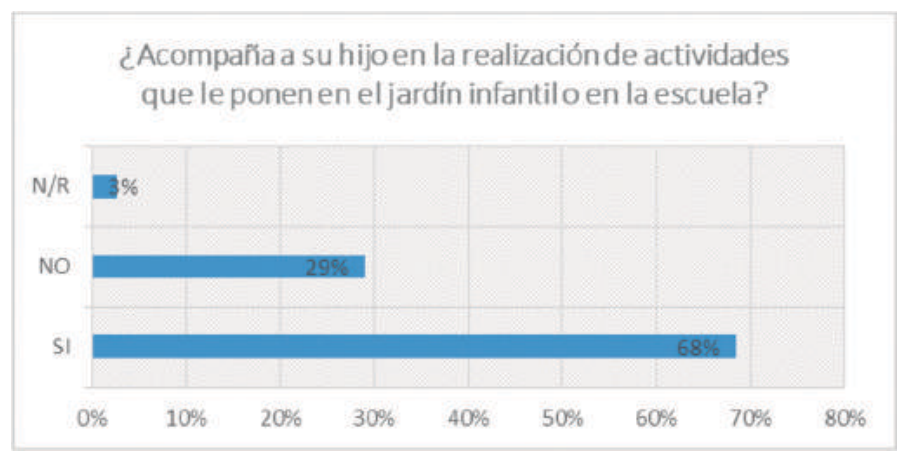

Fuente: elaboración propia.

Figura 3. Acompañamiento en la realización de actividades del jardín infantil o en la escuela

\section{Corporal}

El acompañamiento que realiza la madre adolescente para promover el desarrollo corporal de su hijo permite evidenciar el reconocimiento que hace desde el cuerpo del niño, la construcción de su identidad y las posibilidades de relación que pueda establecer con el medio natural, social y cultural. En la subcategoría de desarrollo corporal, se formularon diferentes preguntas con relación a las acciones que las madres adolescentes realizan para promoverla. La primera de ellas: ¿El niño realiza actividades físicas?, en la cual se evidencia que el 87\% de las madres expresaron que sus hijos SÍ realizan actividad física como: correr, bailar, jugar fútbol, natación, montar bicicleta, entre otras; el 8\% NO realizan actividades físicas, y el 5\% no respondieron a la pregunta. En la pregunta dos: ¿estimuló físicamente al niño recién nacido?, se encuentra que, en su totalidad, 100\% expresaron que SÍ estimularon al niño en sus primeros días de vida, por medio de masajes, movimientos y juegos, lo cual es pertinente para garantizar un desarrollo integral.Y, en la tercera pregunta: ¿el niño se encuentra en programas de control y desarrollo?, el 89\% manifestaron que Sí asisten a programas de control y desarrollo, donde su mayoría refieren haber ingresado al niño entre los 0 a 1 año; el 8\% NO asisten a ningún programa; y el $3 \%$ no respondieron a la pregunta (ver Figura 4). 
Promoción del desarrollo como práctica de crianza de las madres adolescente

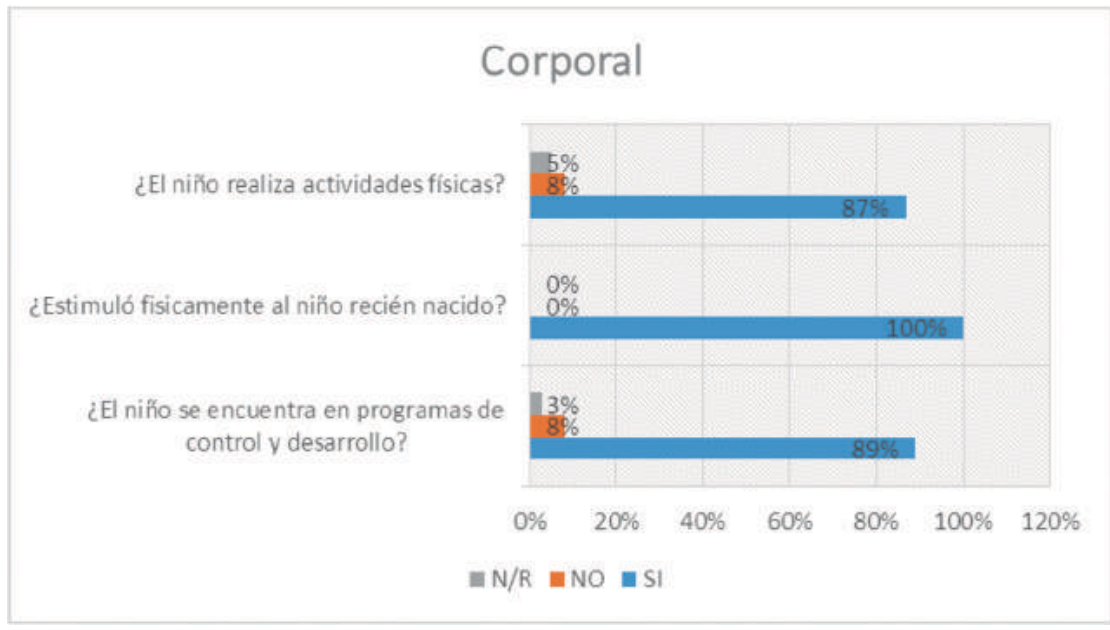

Fuente: elaboración propia.

Tabla 4. Acciones enfocadas a promover el desarrollo corporal

A parte de las actividades físicas, la estimulación y la participación en los programas de crecimiento y desarrollo, se indagó por los juguetes que utilizan sus hijos en la casa o en otros espacios que favorecen su desarrollo corporal. Se evidenció que el 11\% utilizan juguetes como: balones, lazos, juegos de movimiento y triciclos; los demás porcentajes se distribuyen entre la selección de uno o dos juguetes. Y un 5\% manifiestan utilizar otros juguetes como: bicicleta, juegos musicales, bloques lógicos, rompecabezas, entre otros (ver Figura 5). 


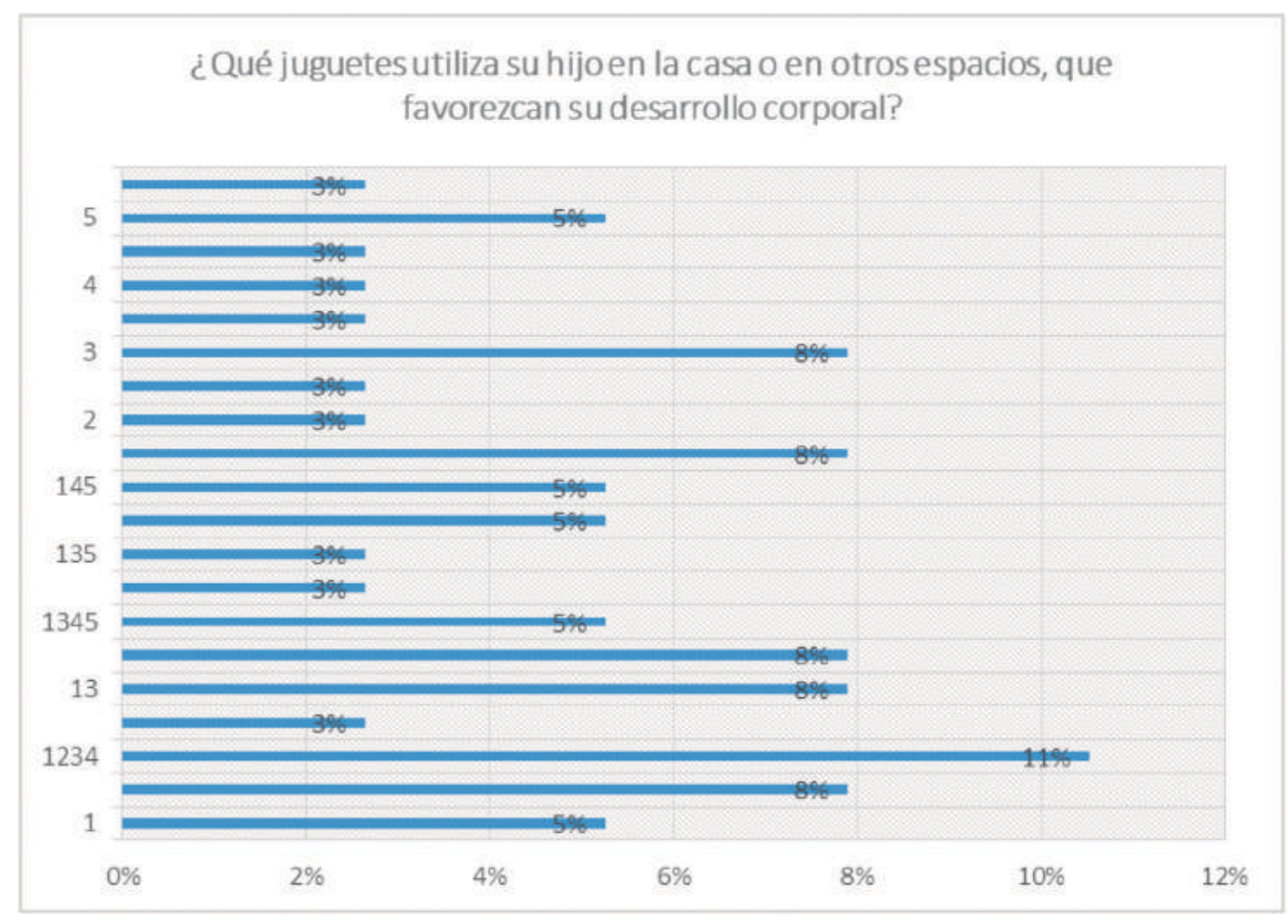

Fuente: elaboración propia.

Figura 5. Juguetes utilizados por el niño en la casa o en otros espacios, que favorezcan su desarrollo corporal. (1) balones, (2) lazos, (3) juegos de movimiento, (4) triciclos, (5) otros.

\section{Estético}

Con respecto a las prácticas de crianza enfocadas a promover el desarrollo estético, es importante resaltar que las madres generan espacios para expresar gustos, estimular lo artístico, y el cuidado del propio cuerpo. Se indagó, por tanto, sobre tres asuntos: primero, si la madre indaga sobre los gustos y sentires del niño. Se logró evidenciar que el 87\% de las madres Sí lo hacen por medio de: preguntas, mostrando opciones; el 8\% de ellas NO lo hacen y el 5\% no respondieron a la pregunta. Segundo, si estimula el desarrollo artístico del niño, se observa que el 89\% de las madres afirman que Sí y el 11\% de ellas afirman que NO estimulan el desarrollo artístico. Y, tercero, si estimula al niño para el cuidado de su cuerpo y sus pertenencias, a lo cual el 92\% de las madres respondieron que Sí, el 3\% que NO y un 5\% no respondieron (ver Figura 6). 
Promoción del desarrollo como práctica de crianza de las madres adolescente

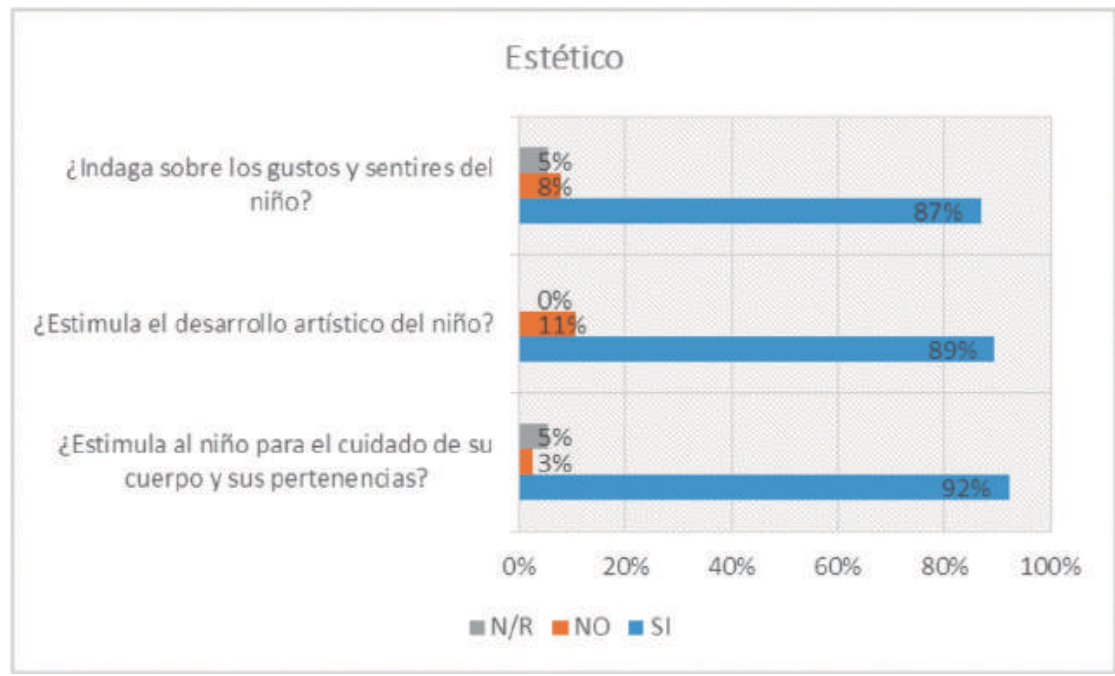

Fuente: elaboración propia.

Figura 6. Acciones enfocadas a promover el desarrollo estético

\section{Discusión}

Las prácticas de crianza implementadas por las madres adolescentes están enfocadas en promover el desarrollo cognitivo, corporal y estético de su hijo, reflejando lo planteado por Aguirre (2000), cuando plantea que las prácticas de crianza son acciones que los padres o cuidadores desarrollan para garantizar el crecimiento y desarrollo psicológico y social de los niños, en favor de su supervivencia y aprendizajes. Acciones que se ven influenciadas por factores sociales, económicos, culturales, educativos, familiares y personales, de los cuidadores a cargo de la crianza de los niños.

Respecto al desarrollo cognitivo, el cual está enfocado en la construcción de conocimientos de los sujetos, se evidencia que en las acciones que las madres adolescentes implementan para promover dicho desarrollo, reconocen la importancia de implementar actividades como: juegos, lecturas, enseñar nociones matemáticas, entre otras, que permitan favorecer el desarrollo de la dimensión cognitiva desde los primeros años de vida, ya que estas posibilitan la movilización de aprendizajes desde una participación activa de los niños en dichos procesos. Respecto a lo anterior, el Ministerio de Educación Nacional -MEN- (2014), plantea la necesidad de facilitar el desarrollo a partir del establecimiento de relaciones tanto en el núcleo familiar, como en la escuela, fundamentales para consolidar los procesos cognitivos básicos: percepción, atención y memoria. 
Por lo tanto, la familia se convierte en un espacio importante para garantizar la construcción de conocimientos, a través de estímulos asociados a la cultura y al ambiente social, los cuales permiten el desarrollo cognitivo a través de los sentidos.

El desarrollo corporal de los niños se promueve a partir del reconocimiento que hacen las madres adolescentes respecto a la estimulación física, la participación en los programas de control y desarrollo promovidos por las entidades prestadoras de salud, la utilización de juguetes pertinentes para la estimulación de la dimensión corporal y la realización de actividades físicas.

Es importen reconocer que los niños son activos por naturaleza, es decir, su cuerpo debe estar en constante movimiento a partir de la realización de actividades físicas y el contacto con objetos, los cuales posibilitan el desarrollo no solo corporal, sino que también ayudan las relaciones interpersonales, teniendo en cuenta que la mayoría de los niños se encuentran en un rango de edad de los 4 a los 6 años, por lo tanto se dan "condiciones de realizar actividades sensoriales y de coordinación de manera mucho más rápida y precisa” (MEN, 2014, p. 18), lo cual posibilita la expresión por medio del movimiento de su cuerpo, la construcción de su personalidad e identidad y la oportunidad para relacionarse con otros sujetos y objetos que hacen parte del mundo que habita.

Con respecto al desarrollo estético del niño, se identifica que está mediado por el contexto cultural, social y familiar en el cual ejerce la crianza la madre adolescente, lo que posibilita la construcción de capacidades en los niños, que les permitan comprender, expresar y transformar las formas en que se percibe el mundo, y cómo se perciben a sí mismos. El cuidador, en este caso la madre adolescente, genera espacios para indagar sobre gustos y sentires, estimular el desarrollo artístico del niño y el cuidado de su cuerpo y sus pertenencias. Esto permite, según lo planteado por el MEN (2014), posibilitar la expresión de emociones, sentimientos y valoraciones que hace el niño de su entorno, lo que ayuda a que se ame a sí mismo y ame a los demás, elementos indispensables para el desarrollo de actitudes de pertenencia, autorregulación y confianza.

Es evidente que las madres adolescentes reconocen la importancia de propiciar espacios para el desarrollo de la dimensión estética, permitiendo que el niño valore sus formas de ser, pensar, sentir y relacionarse con los demás. 
Promoción del desarrollo como práctica de crianza de las madres adolescente

\section{Conclusión}

En conclusión, la mujer en su rol de madre se convierte en una facilitadora del desarrollo de los niños a través de estímulos asociados a la cultura y al ambiente social, que para el caso de las madres del Tecnológico de Antioquia es un ejercicio de responsabilidad, en el cual se propician cuidados físicos y emocionales al niño a través del suministro de la alimentación, la promoción de hábitos y rutinas para la incorporación de normas y comportamientos, y el intercambio de expresiones de afectos verbales y gestuales que fortalecen la creación del vínculo afectivo.

De otra parte, las madres implementan juegos, lecturas, la enseñanza de nociones matemáticas, entre otros, que permiten favorecer el desarrollo de la dimensión cognitiva desde los primeros años de vida, ya que estas posibilitan la movilización de aprendizajes desde una participación activa de los niños en dichos procesos. La dimensión corporal, de igual manera, es promovida por la estimulación física, la participación en los programas de control y desarrollo de las entidades prestadoras de salud, la utilización de juguetes y la realización de actividades físicas.

Sin embargo, para que lo anterior pueda darse, es necesario revisar los factores internos y externos asociados a la crianza, dadas las repercusiones que pueden tener, bien como factores protectores o de riesgo para alcanzar el desarrollo de los niños. Para ilustrar lo dicho, será necesario revisar las condiciones socioeconómicas, si la familia accede a los mínimos vitales de agua potable, alimentación, educación; si viven violencias sociales y familiares, si la madre cuenta con redes de apoyo que la acompañen en el cuidado del niño, el nivel de conocimientos que tienen las madres y sus cuidadores y cómo estos van en coherencia con las prácticas que promueven dicho desarrollo. Así mismo, será necesario considerar si la responsabilidad de la crianza debiera distribuirse en diferentes corresponsabilidades tales como la paterna, las institucionales, las mismas políticas públicas y no dejarla solo a la mujer, tal como las madres de esta investigación lo dejan ver.

\section{Referencias}

Aguirre, E. (2000). Socialización y prácticas de crianza. En E. Aguirre y E. Durán (Eds.), Socialización: prácticas de crianza y cuidado de la salud (pp. 17-92). Bogotá, Colombia: CESUniversidad Nacional de Colombia.

Alvear, C. y Erazo, H. (2003). Adolescentes hablan de paternidad y maternidad en Cartagena de Indias: casos Santa Lucía y Ceballos. Revista Palobra, 7, 92-107.

Amar, J. (2015). Desarrollo infantil y prácticas de cuidado. Barranquilla, Colombia: Editorial Universidad del Norte.

Ander-Egg, E. (1987). Técnicas de Investigación Social. Buenos Aires, Argentina: Magisterio del Río de la Plata. 
CEPAL, Naciones Unidas y OIJ. (2007). La juventud en Iberoamérica. Tendencias y urgencias. Santiago de Chile, Chile: CEPAL, Naciones Unidas.

García, B. y Quintanal, J. (2007). Métodos de investigación y diagnóstico en Educación. Mide. Madrid, España: Módulos de investigación del Centro de Enseñanza Superior “Don Bosco”.

García, T. (2003). El cuestionario como instrumento de investigación/evaluación. En T.García (Ed.), Módulo Etapas del Proceso de Investigación: Instrumentación (pp. 1-29). Madrid, España: UNED.

Henao, N. y Villa, M. (2017). Prácticas de crianza en madres adolescentes: una aproximación a sus concepciones. En C. Córdova y C. Vásquez (Eds.), La educación que necesitamos para el mundo que queremos (pp. 417-428). Guanajuato, México: Universidad De La Salle Bajío.

Hurtado de Barrera, J. (2000). Metodología de la Investigación Holística. Caracas, Venezuela: Sypal.

Ley 1098 de 2006 (08 de noviembre). Por la cual se expide el Código de la Infancia y la Adolescencia. Diaro Oficial No. 46446.

Martínez, J. (2014). Desarrollo infantil: una revisión. Investigaciones Andina, 16 (29), 1118 1137.

Ministerio de Educación Nacional -MEN-. (2014). Lineamientos Curriculares para el Preescolar. Recuperado de https://www.mineducacion.gov.co/1759/articles-339975_recurso_11.pdf

Profamilia. (2010). Fecundidad. Encuesta Nacional de Demografía y Salud 2010. Recuperado de https:/ / dhsprogram.com/pubs/pdf/FR246/FR246.pdf

Ramírez, M. y Barrios, M. (2016). Maternidades y paternidades: Discusiones contemporáneas. Bogotá, Colombia: Universidad Nacional de Colombia.

Restrepo, G. (2015). La neuropsicología transaccional, hacia una concepción integral de las dificultades del desarrollo infantil. Revista Ciencias de la Salud, 13 (3), 431-455.

Torres, M. (2009). Prácticas de crianza y educación inicial en niños Mayo / Yoreme (tesis de maestría). Centro de Investigación en Alimentación y Desarrollo A.C. Hermosillo, Sonora, México.

UNFPA. (2013). Maternidad en la niñez. Enfrentar el reto del embarazo en adolescentes. Recuperado de https: / / www.unfpa.org/sites/default/files/pub-pdf/ES-SWOP2013.pdf

UNICEF. (2011). Estado Mundial de la Infancia 2011. La adolescencia: una época de oportunidades. Recuperado de https://www.unicef.org/honduras/Estado_mundial_infancia_2011.pdf

UNICEF-Centro de Investigaciones Innocenti. (2006). Observaciones Generales del Comité de los Derechos del Niño. Siena, Italia: UNICEF. 